\title{
Chopart's Fracture-Dislocation: A Rare Entity
}

\author{
Hicham Aitbenali, Hamza Madani", Jaouad Yasser, Hamza Benmoussa, Mohammed Shimi
}

Department of Orthopaedics and Traumatology, University Hospital Center of Tangier, Faculty of Medicine and Pharmacy of Tangier, Abdelmalek Essaâdi University, Tangier, Morocco

DOI: $10.36347 /$ sjmcr.2020.v08i06.012

| Received: 01.06.2020 | Accepted: 09.06.2020 | Published: 24.06.2020

*Corresponding author: Hamza Madani

\section{Abstract}

The midtarsal joint's dislocation (Chopart) are uncommon. Dorsal displacement is certainly more uncommon. Osteochondral fractures are frequently associated with these dislocations as has been described in the literature. To the best of our knowledge, the plantarflexion of the forefoot on the dorsiflexed hindfoot with the ankle in a neutral position is behind of dorsal midtarsal dislocation. In this article, we report a rare case of Chopart's dorsal dislocation, with open calcaneal and talus fractures, ruptured tendon. We discuss the dislocation's mechanisms and its treatment. An open reduction was realized with fixation Kirchners wires (K-wires). Moreover, repair of ligamentous structures and immobilization with plaster cast boot.

Keywords: Dislocation, Chopart, Fracture, Open.

Copyright @ 2020: This is an open-access article distributed under the terms of the Creative Commons Attribution license which permits unrestricted use, distribution, and reproduction in any medium for non-commercial use (NonCommercial, or CC-BY-NC) provided the original author and source are credited.

\section{INTRODUCTION}

The Chopart joint, otherwise, transverse tarsal joint was termed after Francois Chopart who performed an amputation at this level [1]; it is composed of 2 articulations: the calcaneocuboid and the talonavicular joint so connecting the hindfoot and midfoot. Damages of the Chopart joint including fractures and dislocations are scarce and are most serious. These injuries are missed or not adequately diagnosed in up to $41 \%$ of cases [2]. They are most caused commonly by highenergy accidents (motor vehicle, fall from height). In this article, we report a unique case of Chopart dislocation associated with a fracture.

\section{Case Report}

An 18 -year-old woman has fallen of approximately ten meters (4th floor) with direct reception on both feet, causing pain with total functional impotence of the left foot. When she was admitted to the emergency department, the patient was conscious, hemodynamically and respiratory stable, she had a swelling on the dorsal aspect of the right forefoot with stage II skin opening according to Couchoix Duparc's classification (figure 1), absence of flexion of the toes and no vascular-nervous deficit.
X-ray foot was taken (figure2), showed dorsal midtarsal dislocation associed with a comminuted fracture of the calcaneus and talus.

The patient was admitted to the operating room; under spinal anesthesia, a careful debridement was carried out. Emergency closed manipulation and reduction done. However, the reduction was unstable. Based on the degree of displacement and disruption of ligamentous structures, we proceeded with open reduction of midtarsal joint. Reduction checked under C-arm (figure 3). Reduction maintained using $3 \mathrm{~K}$ wires(20/10), (2 K-wires transfixing the talonavicular joint in a crossed pattern, $1 \mathrm{~K}$-wire for talus fracture fixation, and $1 \mathrm{~K}$-wire for calcaneal fracture fixation ). Identified torn ligaments and capsule repaired. Following the operation, the right foot was immobilized in a short-leg cast for 6 weeks after the plaster has been removed; the patient has benefited from functional rehabilitation for analgesic purpose and recovery of the joint amplitudes. 


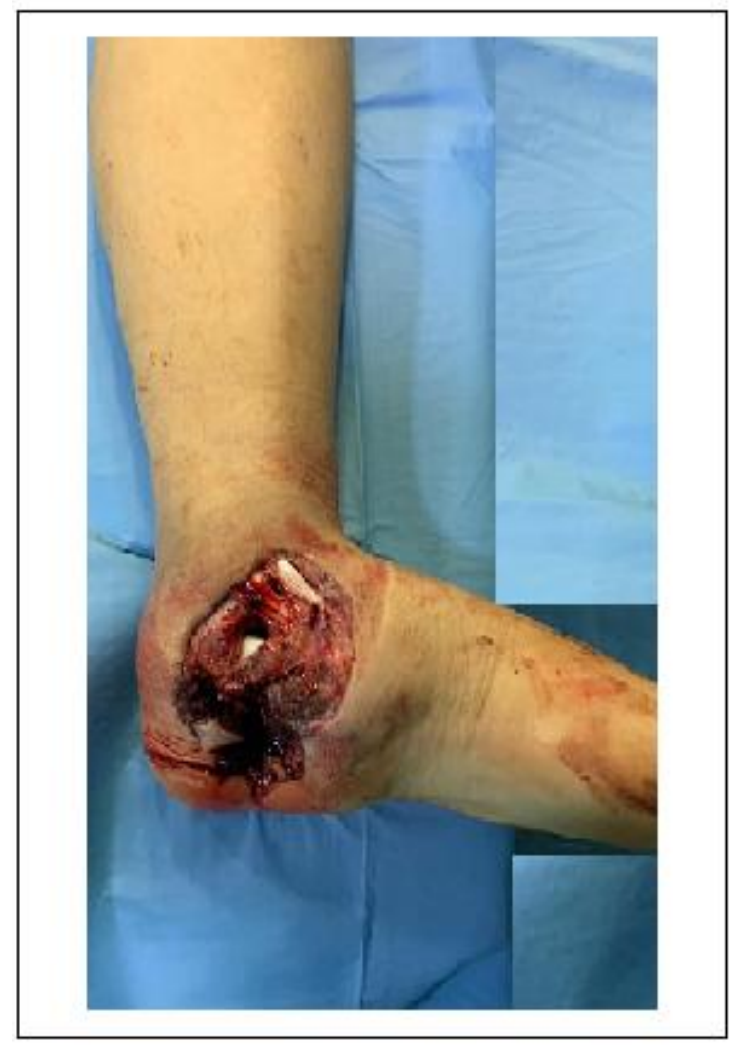

Fig-1: Skin opening stage II COUCHOIX DUPARC

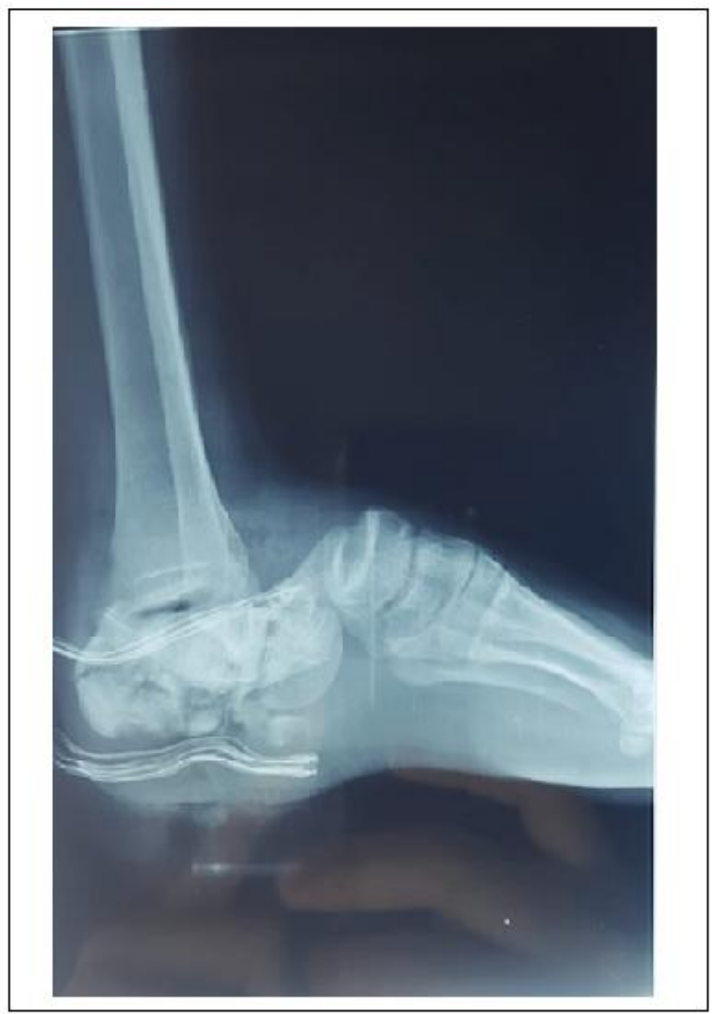

Fig-2: Strict profile incidence showing dorsal Chopart dislocation with comminuted calcaneus and talus fracture

\section{DISCUSSION}

The anatomy of midtarsal joint complex, namely surrounding bone articulations and ligament structures of the cuboid and navicular bones, make it stable, therefore, it requires very high energy to dislocate, and this dislocation is likely to result in fractures of the peripheral talus, navicular, cuboid, and calcaneus. These lesions are serious and may cause disabling conditions [3]. This is the case of our patient who is admitted for a dorsal dislocation of the Chopart joint associated with fractures of the calcaneus and talus following a high-energy trauma.

Dorsal midtarsal dislocation is rare. The mechanism of their occurrence is a trauma in forced dorsal flexion with a rupture of the plantar, talocalcaneal and calcaneocuboid union's means, which are very resistant. In this case, the search for compression lesions on the talar head and / or the dorsal area of the calcaneocuboid is essential [4]. In our observation, we believe that the fall from a height of about $10 \mathrm{~m}$ on the sole of the foot in forced dorsal hyper flexion probably damaged the midfoot's ligaments and consequently, a dorsal fracture-dislocation occurred, this can also explain the cutaneous opening and tendon ruptures, especially since the trauma has been severe. To our knowledge, three cases of isolated dorsal dislocation have been published in the literature [5-7], two cases of fracture-dislocation have been reported [8] were treated by open reduction and internal fixation with k-wires. However, no case of open fracturedislocation has been reported.

The main aim of treatment is to correct the anatomical orientation of the foot by immediate reduction and stabilization of the dislocation. Closed reduction is privileged. In case of impaction causing malalignment, an open reduction must be carried out by the use of either Kirschner wires or Schanz screws, which are placed in the cuneiforms, the calcaneus and the talus [3].

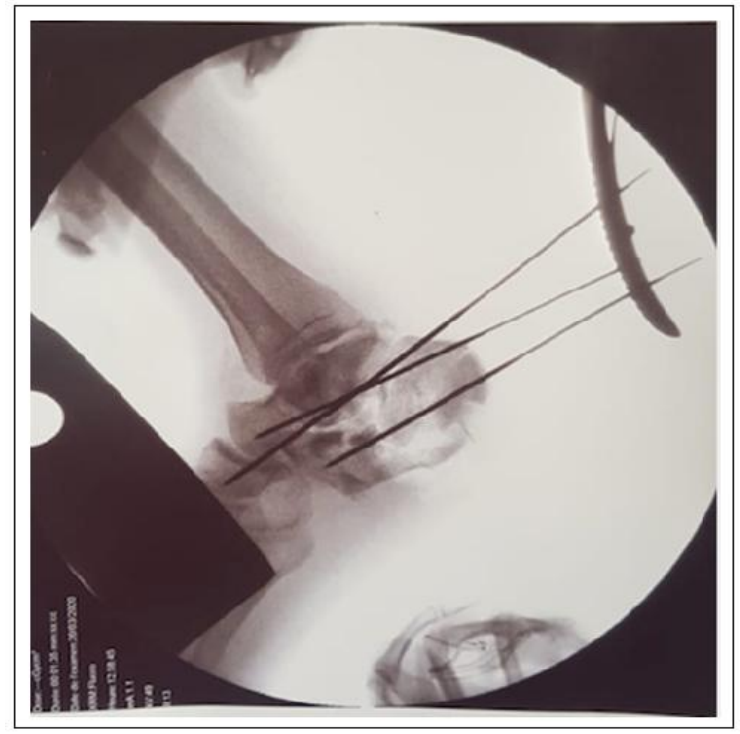

Fig-3: Reduction checked under $\mathrm{C}$-arm reduction of dislocation and fractures and stabilization by three kirschner wires (K-wires) 
The antero-lateral and antero-medial approaches allow better visualization of the midtarsal joint.

Open fracture-dislocation is a surgical emergency. Treatment should include debridement of the wound, joint washing, reduction and stabilization of the dislocation by $\mathrm{K}$-wires and repair of associated lesions. In case of high septic risk, an external tibiopedal fixator makes stabilization [4].

The long-term development is marked by the risk of developing post-traumatic arthritis, case where arthrodesis remains the most suitable treatment.

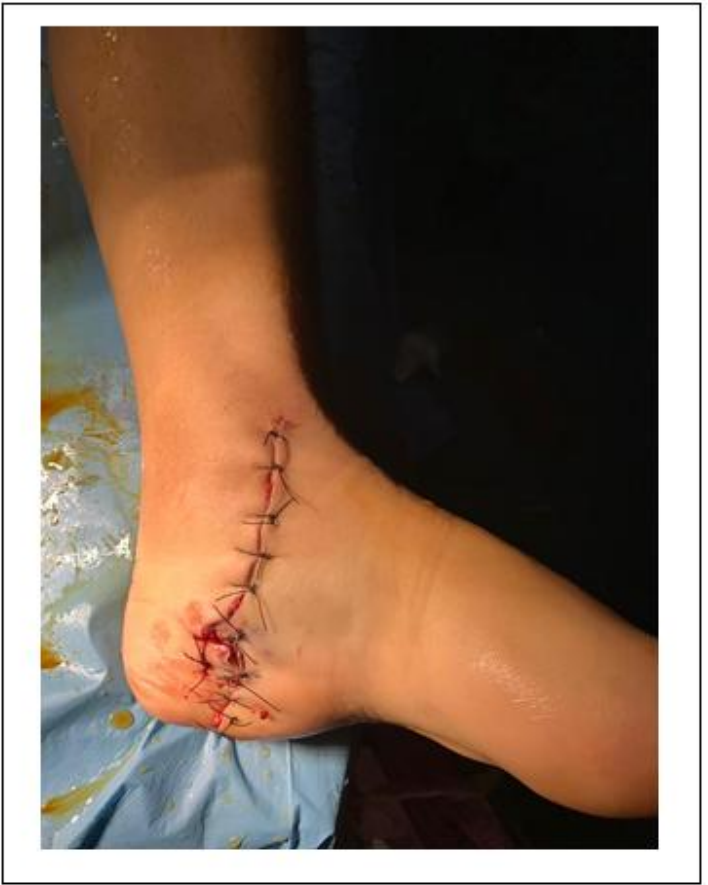

Fig-4: wound suturing

\section{CONCLUSION}

The Chopart fracture dislocation is a rare entity. Reduction and stabilization are to be performed in emergency. The evolution is especially marked by the risk of occurrence of post-traumatic osteoarthritis.

\section{REFERENCES}

1. Honeycutt MW, Perry MD. The Chopart Variant Dislocation: Plantar Dislocation of the Cuboid and Navicular. Foot \& Ankle Orthopaedics. 2019 Sep 16;4(3):2473011419876262.

2. Van Dorp KB, de Vries MR, van der Elst M, Schepers T. Chopart joint injury: a study of outcome and morbidity. The Journal of foot and ankle surgery. 2010 Nov 1;49(6):541-5.

3. Klaue K. Treatment of Chopart fracturedislocations. European Journal of Trauma and Emergency Surgery. 2010 Jun 1;36(3):191-5.

4. Biga N. Chirurgie des traumatismes du pied et de la cheville: Lésions récentes et anciennes de l'adulte. Elsevier Masson; 2010 Nov 17.

5. Ip KY, Lui TH. Isolated dorsal midtarsal (Chopart) dislocation: a case report. Journal of Orthopaedic Surgery. 2006 Dec;14(3):357-9.

6. Puthezhath K, Veluthedath R, Kumaran CM, Patinharayil G. Acute isolated dorsal midtarsal (Chopart's) dislocation: a case report. The Journal of foot and ankle surgery. 2009 Jul 1;48(4):462-5.

7. Chbani B, Amar MF, Lahrach K, Marzouki A, Boutayeb F. Luxation dorsale médiotarsienne (Chopart) isolée. Médecine et chirurgie du pied. 2010 Oct 1;26(3):81-3.

8. Khan AP, Raffic M. Chopart's Fracture-dislocationrare Cases Management. Kerala Journal of Orthopaedics. 2018 Jan 1;31(1). 